\title{
KOMMENTAR
}

\section{Deflationäre Stagnation abwenden!}

Das vergangene Jahr brachte die schwerste globale ökonomische Krise seit der Weltwirtschaftskrise Ende der 1920er, Anfang der 30er Jahre. Obwohl der Abschwung vorerst gestoppt scheint, ist eine Rückkehr zum Wachstumspfad vor der Krise in weiter Ferne. Vielmehr droht weiterhin eine deflationäre Stagnation - eine Gefahr, die von der Wirtschaftspolitik und großen Teilen der wissenschaftlichen Politikberatung hartnäckig ignoriert wird.

Die gegenwärtige Krise markiert einen Strukturbruch in der längerfristigen wirtschaftlichen Entwicklung. Diese war seit den 1980er Jahren durch das ,neoliberale“ oder finanzdominierte Entwicklungsmodell der Deregulierung der Arbeitsmärkte, des Abbaus (sozial)staatlicher Eingriffe in den Wirtschaftsprozess, der Umverteilung von den (unteren) Arbeitseinkommen zu den hohen Managergehältern und den Gewinneinkommen sowie der Deregulierung der nationalen und internationalen Finanzmärkte geprägt. Dieses Modell hat in einigen entwickelten Industrieländern, z. B. in den USA und Großbritannien, in Kombination mit der dort praktizierten expansiven makroökonomischen Politik zu hohen Wachstumsraten und geringer Arbeitslosigkeit geführt. Und es hat auch für die Weltwirtschaft insgesamt, mit den USA als Konjunkturlokomotive, zu einer langen Phase der Prosperität beigetragen.

Allerdings war das Modell des finanzdominierten oder „neoliberalen" Kapitalismus durch zunehmende, letztlich nicht aufrecht zu erhaltende Ungleichgewichte gekennzeichnet. Zum einen bedeutete die zunehmende Ungleichheit in der Einkommensverteilung, dass die dynamische, die Weltkonjunktur tragende Konsumnachfrage in den USA nur durch eine zunehmende Verschuldung der privaten Haushalte vor dem Hintergrund erst des Aktienpreis- und dann des Immobilienpreisbooms ermöglicht wurde. Diese latente Überschuldung der privaten Haushalte war dann zusammen mit einem leichten Zinsanstieg in den USA der Auslöser der Finanzkrise. Zum anderen baute die Konjunkturlokomotive USA immer größere Leistungsbilanzdefizite mit einem entsprechend wachsenden Bedarf an Kapitalimporten zur Finanzierung auf, denen entsprechende Leistungsbilanzüberschüsse und damit Kapitalexporte in China, Deutschland und Japan gegenüberstanden. Durch die Kapitalexporte nahm zum einen die Ansteckungsgefahr der Überschussländer in einer Finanzkrise zu, zum anderen waren diese Länder wegen ihrer übermäßigen Exportabhängigkeit besonders vom Rückgang der Weltkonjunktur in der Krise betroffen. Zusammen mit den Regulierungsdefiziten auf den Finanzmärkten waren damit die Ungleichheiten und Ungleichgewichte für die Tiefe und die rasche Verbreitung der Finanz- und Wirtschafskrise über den Globus verantwortlich.

Als Ergebnis werden die USA auch nach der Krise als Weltkonjunkturlokomotive ausfallen. Die US-Haushalte haben ihre Sparquoten bereits deutlich erhöht und werden dies weiterhin tun. Und die öffentlichen Haushaltsdefizite werden allein schon aus politischen Gründen nicht auf hohem Niveau verbleiben. Neben einer grundlegenden Re-Regulierung des Finanzsektors, der auf seine wesentliche makroökonomische Aufgabe - die Finanzierung von realen Investitionen - zurückgeführt werden muss, ist daher eine dauerhafte Neuausrichtung der makroökonomischen Politik vor allem in den bisherigen Überschussländern erforderlich.

Dies schließt für Deutschland und die Europäische Währungsunion erstens eine mittelfristige Festlegung der Europäischen Zentralbank (EZB) auf eine die Realwirtschaft stützende Politik der niedrigen Zinssätze ein. Zweitens ist seitens der Lohnpolitik gerade in der Krise alles zu unternehmen, um deflationäre Impulse zu vermeiden. Nur wenn es gelingt, ein Nominallohnwachstum in Höhe der Summe aus mittelfristigem Produktivitätswachstum und Zielinflationsrate durchzusetzen, wird die Lohnpolitik letztlich Beschäftigung sichernd sein. Drittens sollte die Fiskalpolitik auch mittelfristig expansiv ausgerichtet bleiben, um ein hohes Niveau der gesamtwirtschaftlichen Nachfrage zu gewährleisten insbesondere durch eine auch angebotsseitig gebotene Ausdehnung der öffentlichen Investitionen im weiteren Sinne. Zudem ist die internationale Koordination eines solchen expansiven makroökonomischen Politik-Mixes, zumindest im Euroraum, erforderlich.

Wirft man jedoch einen Blick auf die mittelfristige Ausrichtung der Wirtschaftspolitik, so wird sie den mit der Krise und dem Strukturbruch aufgeworfenen Problemen kaum gerecht. Die EZB scheint mehr mit vermeintlichen zukünftigen Inflationsgefahren als mit den real existierenden Deflationsgefahren beschäftigt zu sein. Die deutsche Lohnpolitik sieht sich den Forderungen nach einer angeblich Beschäftigung sichernden Lohnzurückhaltung ausgesetzt, die das Deflationsrisiko weiter erhöht und die neomerkantilistische Lohndumpingpolitik fortzusetzen droht, die schon bisher einen gefährlichen Sprengsatz für die Währungsunion darstellt. Und die Fiskalpolitik droht angesichts der makroökonomisch irrationalen grundgesetzlichen „Schuldenbremse“ äußerst restriktiv zu werden. Zudem war Deutschland als wirtschaftlich bedeutendstes Land der Währungsunion in der Vergangenheit mehr ein Verhinderer denn ein Gestalter eines koordinierten internationalen makroökonomischen Gegensteuerns gegen die Krise. Gelingt es nicht, diese Tendenzen der Wirtschaftspolitik zu überwinden, so wird es schwer, eine deflationäre Stagnation zu vermeiden.

\footnotetext{
Prof. Dr. Eckhard Hein, Hochschule für Wirtschaft und Recht Berlin und Institute for International Political Economy (IPE) Berlin. Arbeitsschwerpunkte: Makroökonomie, Finanzsystem und wirtschaftliche Entwicklung, Verteilung und Wachstum, europäische Wirtschaftspolitik. e-mail: eckhard.hein@hwr-berlin.de
} 